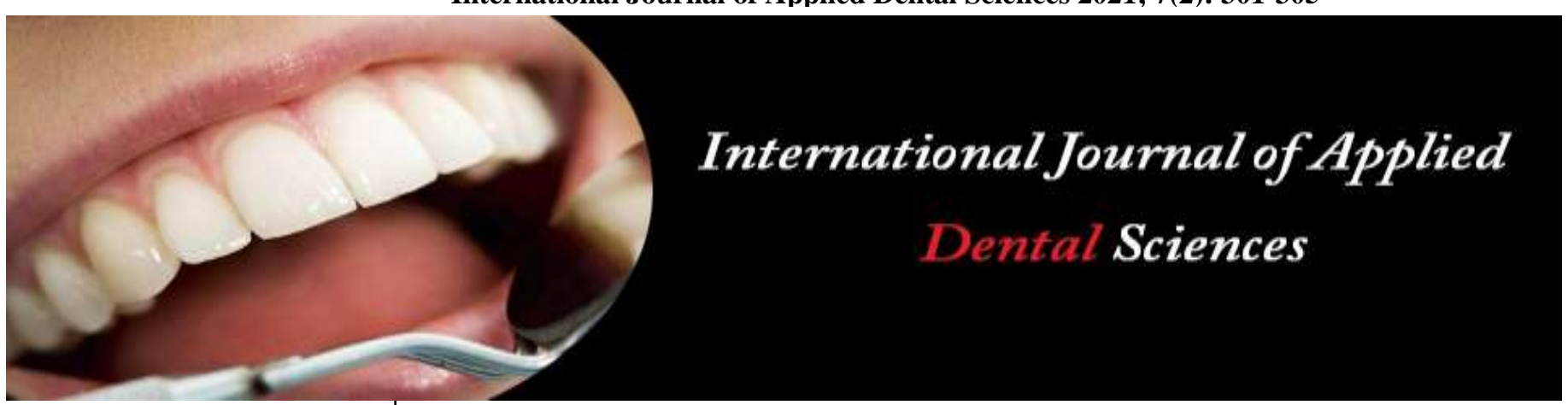

ISSN Print: 2394-7489

ISSN Online: 2394-7497

IJADS 2021; 7(2): 301-305

(C) 2021 IJADS

www.oraljournal.com

Received: 22-01-2021

Accepted: 13-03-2021

Dr. Anoopa Nataraj

Post Graduate Student,

Department of Prosthodontics,

D. Y. Patil University School of

Dentistry, Navi Mumbai,

Maharashtra, India

Dr. Asha M Rathod

Professor, Department of

Prosthodontics, D. Y. Patil

University School of Dentistry,

Navi Mumbai, Maharashtra,

India

Dr. Gaurang Mistry

Head of the Department,

Department of Prosthodontics,

D. Y. Patil University School of

Dentistry, Navi Mumbai,

Maharashtra, India

Dr. Ashwini Kini

Associate Professor, Department of Prosthodontics, D. Y. Patil

University School of Dentistry,

Navi Mumbai, Maharashtra,

India

Dr. Sheetal B Parab

Associate Professor, Department

of Prosthodontics, D. Y. Patil

University School of Dentistry,

Navi Mumbai, Maharashtra,

India

\section{Transferring occlusal vertical dimension in full arch implant prosthesis: A review}

\author{
Dr. Anoopa Nataraj, Dr. Asha M Rathod, Dr. Gaurang Mistry, Dr. \\ Ashwini Kini and Dr. Sheetal B Parab
}

DOI: $\underline{\text { https://doi.org/10.22271/oral.2021.v7.i2e.1223 }}$

\section{Abstract}

The maxillo-mandibular relationship record is a critical step to establish occlusion in implant supported full mouth rehabilitation involving fixed or removable prosthesis. The definitive implant casts have been articulated by several techniques at optimum occlusal vertical dimension (OVD) in the correct centric relation $(\mathrm{CR})$ in edentulous patients receiving implants. Newer methods have been discussed for transferring vertical jaw relation that have claimed to reduce chair side time, establish accurate records and increase comfort for both, the patient and the clinician. The various techniques are, stabilized record bases with occlusal rims screwed in or supported by healing abutments, plastic sprues reinforced by acrylic record base, duplicate denture, relining existing denture, use of buccal index of existing denture and use of silicone cones. Thus, a review of the various techniques available to transfer these records onto the articulator is enlisted, from which the clinician is free to choose the technique most feasible.

Keywords: Occlusal vertical dimension, Implant supported prosthesis, stabilized record base, duplicate denture, healing abutment

\section{Introduction}

Implant supported fixed and removable prosthesis have been successfully used in edentulous patients over conventional complete dentures to improve function, comfort and satisfaction for such patients ${ }^{[1]}$. The prosthodontic protocol for the treatment of fully edentulous patients who receive osseointegrated implants follows the principles of fabricating conventional complete dentures ${ }^{[2]}$. However, several techniques have been adopted over the years by various authors to overcome the challenge of registering \& transferring accurate vertical jaw relation records to the articulator. The correct transfer of maxillo-mandibular relations is essential for providing proper design of prosthesis and ideal occlusion ${ }^{[3,4]}$.

Techniques for mounting the definitive implant cast on the articulator include the usage of:

1) Record bases with occlusal rims

2) Duplicate dentures

3) Using existing dentures

4) Elastomeric record base and facial index of existing denture

Stable acrylic record bases with occlusal rims:

Stable record bases are critical in accurate registration procedures for measuring vertical dimension of occlusion and centric relation in the edentulous patient. The record base must be rigid, accurate and stable to perform the intended functions. ${ }^{[2]}$ Various techniques have been described to secure the record base, such as:

a) Screw retained gold cylinders ${ }^{[5-7]}$

b) Plastic healing caps ${ }^{[8]}$

c) Plastic sprues ${ }^{[9]}$ and

d) Bases supported by healing abutments ${ }^{[2]}$
Dr. Anoopa Nataraj

Post Graduate Student,

Department of Prosthodontics,

D. Y. Patil University School of

Dentistry, Navi Mumbai.

Maharashtra, India 
Screw retained Gold cylinders placed on abutment analogues over implant definitive casts are secured with prosthetic screws (Fig 1). Acrylic resin denture base must attach to each gold cylinder, provide base for wax occlusal rims and must have incisal access for the cylinders to be screwed into the abutments. The record base is entirely implant supported, over which wax occlusal rims are fabricated.
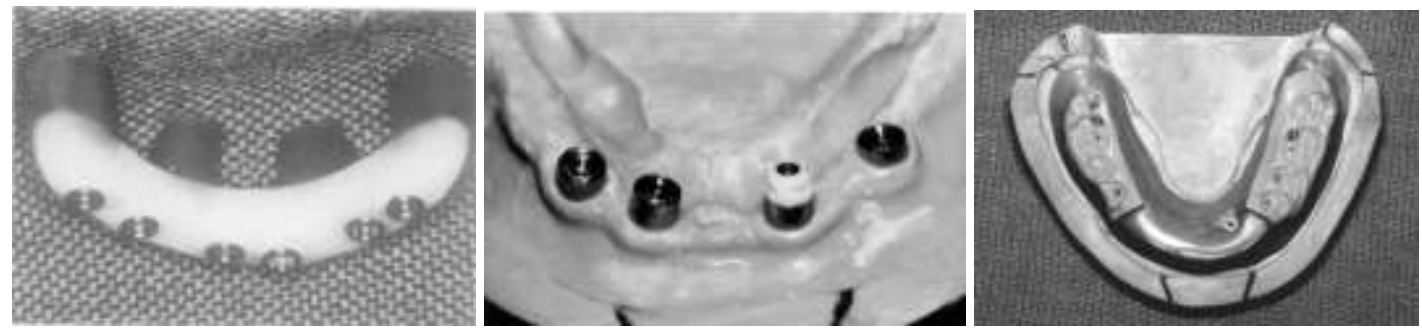

Fig. 1: Left- Gold cylinders attached to resin base and wax blocks; Middle- Modified healing cap attached to master cast; Right- Record base before mounting on an articulator.

A modified plastic healing cap with retention groove (Fig. 1middle) is screwed in over a healing abutment and acrylic resin is adapted over the rest of the healing abutments and any excess resin is cleared off the modified plastic healing cap to provide screw access. The record base with rims can be retrieved by unscrewing the modified healing cap (Fig. 1left).
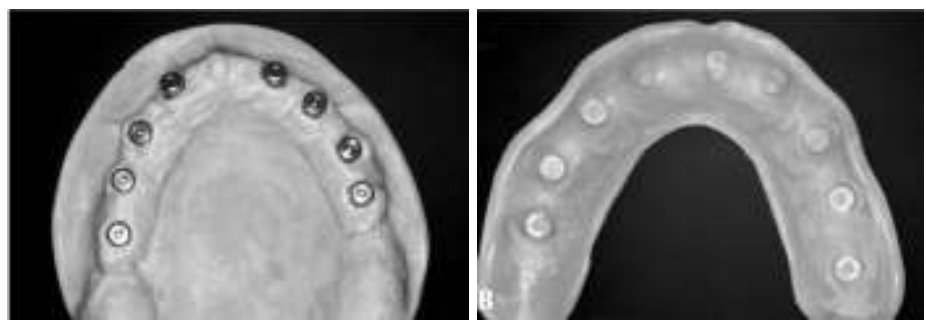

Fig. 2: Left- Healing abutments on master cast; Left- Internal surface of record base showing negative impression of healing abutments. Base extends 5-8mm facially and lingually

Another technique describes a stable record base made over healing abutments (Fig. 2). It describes establishing a stable record base without the necessity of removing the healing abutments. A replica of healing abutments are screwed into the implant analogues on the master cast. Undercuts are then blocked out with blockout putty and a record base made over these healing abutments. This technique provides for convenient adjustment procedure during try in, minimizes patient discomfort as healing abutments are not removed. The only disadvantage of this procedure is the additional cost for the healing abutments on the master cast. Plastic sprues of 14 gauge can be heated over a flame and placed onto implant analogues embedded in the stone cast to conform to the inner part of the implant (Fig. 4). These sprues are to be joined to each other with the help of the same material and reinforced by acrylic resin, over which occlusal rims are made. There is no displacement of record base and soft tissue during interocclusal recording procedure. Breaking of plastic sprues in the implant fixtures can be easily removed with a warm explorer, and is a rare problem.
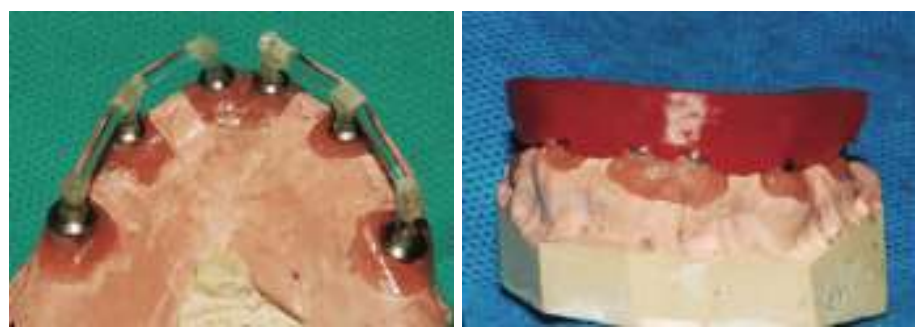

Fig. 3: Left- Plastic sprues over implant analogues; Left- Wax record block made over pattern resin reinforced sprues

However, these approaches demand extra laboratory steps \& is time consuming due to repeated removal and replacement of healing/prosthetic abutments, impression copings or similar components during the clinical appointments ${ }^{[10]}$.

Occlusal rims with record base, once secured intraorally are then adjusted according to the vertical dimension desired and centric is recorded as done for a complete denture jaw relation.

\section{Duplicate denture}

Complete denture acts as a pretreatment prosthesis, which establishes a proper OVD and tooth position before placement of implants and fabrication of the superstructure bar. Such dentures help reestablish or evaluate OVD and also the psychological attitude of a patient before irreversible implant procedures are begun. They can be duplicated to be used as surgical guides and used as interim denture after implant surgery by relining the denture base ${ }^{[3]}$. Once a complete removable denture is esthetically accepted by the patient and OVD and CR are favorable, it can be used as a guide for recording as well as for abutment selection.

The duplicated denture can be made of acrylic resin or Vinyl polysiloxane material. 

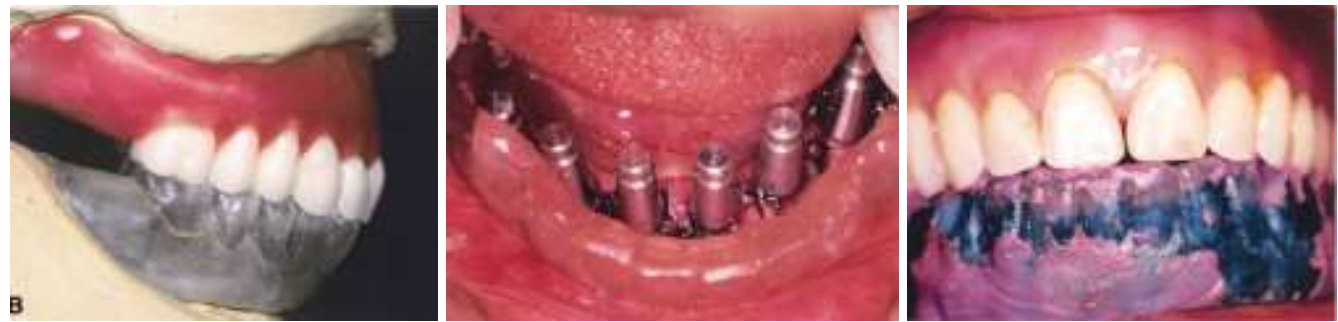

Fig. 4: Left- Clear duplicate mandibular denture; Centre-Duplicate denture modified to form surgical guide for implant placement; Right- Final impression and interocclusal record made simultaneously.

\section{Using acrylic resin}

When made with acrylic resin, this duplicate denture aids to act as a custom tray to make implant/abutment level impressions and also acts as a vertical stop to register the occlusal vertical dimension (Fig. 4). Impression surface of the denture is scrapped and relined with an appropriate impression material. Holes are drilled into the denture corresponding to the position of the healing abutments. This allows for space for the transfer copings which are screwed in $[11,12]$

\section{Using polyvinyl siloxane}

The denture may be duplicated in vinyl polysiloxane material, after being verified intraoally over the healing abutments. It is placed on the implant definitive cast until it snaps into place (Fig. 5). Care must be taken not to apply excessive pressure due to flexible nature of this material. This assembly is then transferred to the articulator with the help of interocclusal records and occlusal vertical dimension re-established ${ }^{[13]}$.

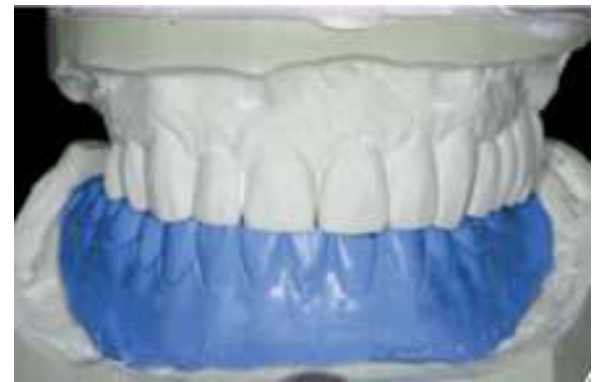

Fig. 5: Maxillary cast articulated Vinyl poly-siloxane denture

\section{Using existing denture}

The existing denture can be converted into an interim fixed prosthesis after verification of OVD intraorally and transferred on the articulator, thus minimizing chair time and increasing patient comfort and satisfaction. This technique allows the clinician and the patient to considerably shorten treatment time. Two methods are described for the same:

a) Relining existing denture

b) Using silicone cones

\section{Relining existing denture}

The intaglio surface of the denture, corresponding to healing abutments is relieved and relined with autopolymerizing resin when the patient occludes at centric relation position. This relined denture is placed on the implant definitive casts with healing abutments in place. Denture flanges are adjusted if it interferes with passive seating of the denture on the cast (Fig. 6). Definitive implant cast and opposing cast are articulated with previously made CR record. Also, a putty index of buccal surface of the interim denture aids in abutment selection and framework design ${ }^{[14]}$.
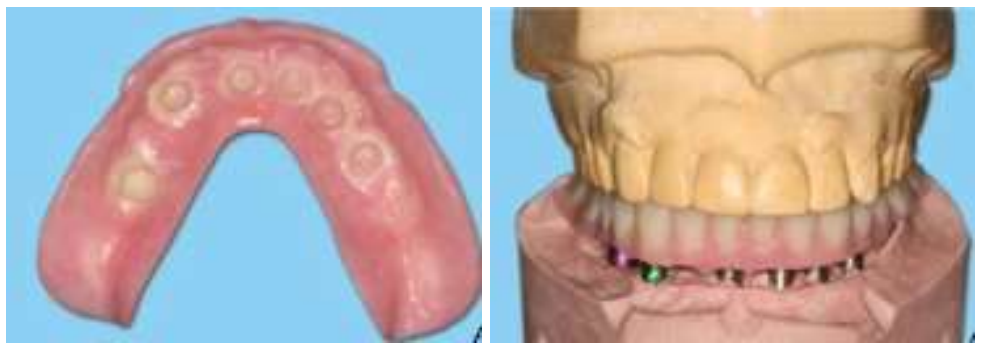

Fig. 6: Left- Intaglio surface of relined lower denture over healing abutments; Right-Casts articulated with existing denture

\section{Using silicone cones}

Another chairside technique to articulate definitive implant cast using the existing denture and silicone cones ${ }^{[1]}$ is described by Parnia et al. Holes are drilled through the artificial teeth of the denture, corresponding to each implant level abutment. Rigid interocclusal material is injected into the holes around the abutment and a CR record is made at the determined vertical dimension (Fig. 7). Transfer the abutments to the mandibular implant cast and place each section of the bite registration material over the corresponding abutment. Thus articulate the mandibular implant cast with the maxillary denture cast with the help of bit registration material segments. The success of this method depends on a well-established denture. When a single arch needs to be restored, opposing arch can be used as reference point. When both arches need to be restored, the opposite denture 
duplicated casts are mounted in the articulator. Each cast is used as reference point for the opposing implant working cast at the same OVD. The accuracy of this method decreases when adequate implants are not place bilaterally. This technique is similar to Cranin ${ }^{[15]}$ and Misch [16], who described the use of plastic template to capture the final denture contour.

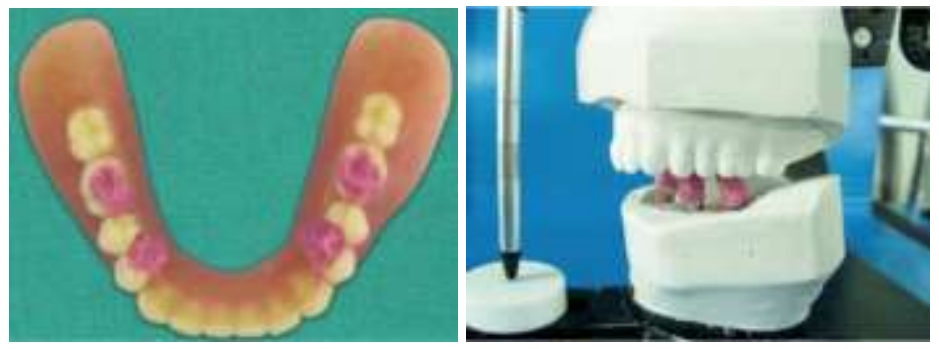

Fig. 7: Left- Interocclusal record at established OVD; Right- Articulation of casts in CR according to silicone cones

The success of this method depends on a well-established denture. This technique is contraindicated when the existing denture does not meet the ideal esthetic, phonetics, vertical dimension of occlusion and centric relation. When a single arch needs to be restored, the opposing arch can be used as a reference point. However, when restoring both arches, the opposite denture duplicated casts of both arches are mounted on the articulator. Each cast is used as the reference point for the opposing implant working cast at the same VDO.

The described procedure saves time, because it eliminates the need to fabricate the recording bases and rims, thus providing a significant comfort for both the patient and clinician. Although, using patients existing denture for recording the jaw relation requires implant definitive cast to be modified extensively to completely seat the denture (with unaltered flanges) on it. This drawback may influence the correct seating of the denture on the implant definitive cast causing faulty recording of the vertical relation ${ }^{[10]}$.

\section{Elastomeric record bases \& Facial index of existing denture}

Existing dentures must be relined after stage II surgery with a hard or soft liner and the jaw relation records verified. Once proved satisfactory, the dentures are joined with sticky wax intraorally. A vacuum formed sheet of $2 \mathrm{~mm}$ thickness is adapted over the facial polished surface of the joined dentures. This forms the facial surface index (FSI). The FSI must be trimmed along the flanges of the denture to make it look like a mouth guard (Fig. 8- left). Acrylic framework is adapted over the healing abutments on the implant definitive cast, which acts as a reinforcement to the elastomeric material used to record intaglio surface and the vertical relation via the facial index. Upper and lower silicone impression is made with the resin framework (Fig. 8-centre). Once in the mouth, silicone putty is adapted over the record bases one at a time until the desired vertical dimension is achieved which can be verified with the FSI in place, intraorally. Once verified, the record is sealed with silicone putty, placed on inner aspect of FSI and carried in the patient's mouth for verification (Fig. 9). Thus, the FSI can be considered an initial guideline to set the OVD. Recording the vertical dimension with FSI alone is not recommended. FSI cannot be repeatable at the same position. The resin framework avoids unnecessary flexion/ distortion of the elastomeric record bases. Although this technique provides accurate records for a single case, further study is required to prove its routine clinical utility ${ }^{[10]}$.
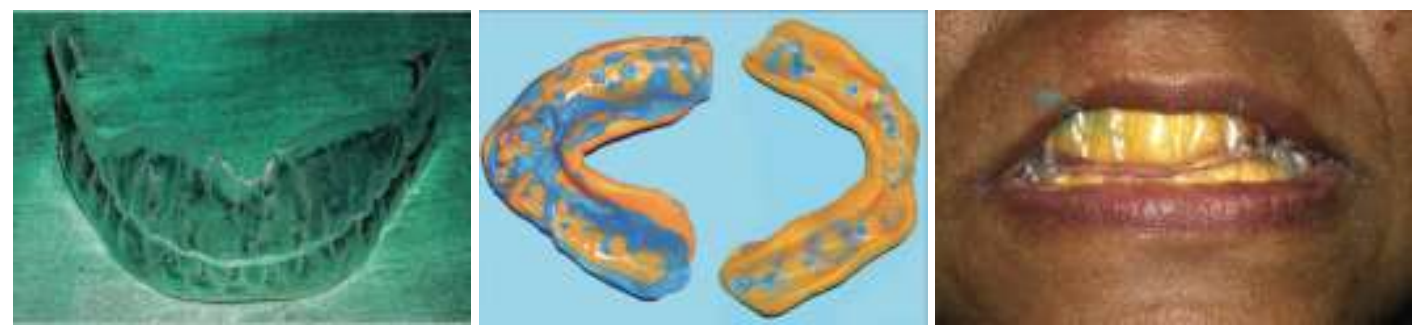

Fig. 8: Left- Facial surface Index; Centre- Reinforced elastomeric record bases; Right-Recording OVD
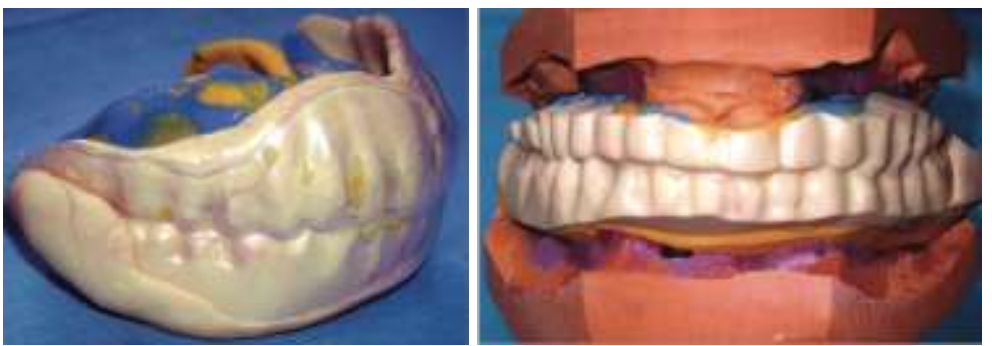

Fig. 9: Left- Completed maxillo mandibular relationship record; Right- Excess flanges cut and implant definitive casts seated

\section{Summary}

This article enlists various techniques to mount the definitive the definitive implant casts at optimal occlusal vertical dimension (OVD) in the correct centric relation (CR) in edentulous patients receiving implants. Clinicians are free to choose any one or more techniques combined, to transfer the vertical and horizontal dimension of occlusion. The importance of the same cannot be overstated, as the long-term 
success of implant fixed restorations lies in accurately reproduced jaw relation records.

\section{References}

1. Parnia F, Moslehifard E, Motayagheni N, Pournasrollah A. A time saving method for Transferring Occlusal Vertical Dimension and Centric Relation of Complete Denture to a Full Arch Implant Prosthesis. J Contemp Dent Pract 2014;15(5):672-75.

2. Rungcharassaeng K, Kan JY. Fabricating a stable record base for completely edentulous patients treated with osseointegrated implants using healing abutments. J Prosthet Dent 1999;81(2):224-7.

3. Misch CE. Dental Implant Prosthetics. St Louis, Mo: Mosby 2005,245-7.

4. Kokubo Y, Ohkubo C. Occlusion recording device for dental implant-supported restorations. J Prosthet Dent 2006;95:262-3.

5. Loos LG. A fixed prosthodontic technique for mandibular osseointegrated titanium implants. J Prosthet Dent 1986;55:232-42

6. Zarb GA, Bolender CL, Carlsson GE. Boucher's prosthodontic treatment for edentulous patients. 11th ed. St Louis: Mosby-Year Book 1997,183-96p.

7. Branemark PI, Zarb GA, Albrektsson T. Tissueintegrated prostheses. 5th ed. Chicago: Quintessence 1985,257-60p.

8. Keith SE, Guillen GE. Enhanced stability for implant-supported overdenture record bases. J Prosthet Dent 1998;79:359-60.

9. Savabi O, Yosefimoghadam A, Nejatidanesh F. A method for making the implant-supported record bases. J Oral Implantol 2009;35:300-2.

10. Patil PG, Nimbalkar-Patil S. Maxillomandibular relationship record for implant complete mouth rehabilitation with elastomeric material and facial surface index of existing denture. J Indian Prosthodont Soc 2015;15:337-41.

11. Rodrigues AH, Morgano SM, Guimaraes MM, Ankly R. Laboratory processed acrylic resin provisional restoration with cast metal substructure for immediately loaded implants. J Prosthet Dent 2003;90:600-4.

12. Daher T, Meserkhani PV, Baba NZ, Morgano SM. Time saving method for the fabrication of ade nitive cast for an implant supported prosthesis. J Prosthet Dent 2007;98(1):70-71.

13. Abbo B, Razzoog ME. Transferring the existing occlusal vertical dimension using a duplicate denture. J Prosthet Dent 2007;98:68-9.

14. Papaspyridakos P, Lal K. Simple technique to transfer occlusal vertical dimension and articulate a de nitive implant cast for a full arch implant prosthesis. J Prosthet Dent 2008;100(4):320-322.

15. Cranin AN, Klien M, Simon A. Atlas of oral implantiology. New York, NY: Thieme Medical Publishers Inc 1993,213-226.

16. Misch CE. Contemporary implant dentistry. 2nd ed. St. Louis, MO: Mosby Inc 1999,588. 